\title{
Poisson automorphisms and quiver moduli
}

\author{
Markus Reineke \\ Fachbereich C - Mathematik \\ Bergische Universität Wuppertal \\ D - 42097 Wuppertal, Germany \\ e-mail: reineke@math.uni-wuppertal.de
}

\begin{abstract}
A factorization formula for certain automorphisms of a Poisson algebra associated with a quiver is proved, which involves framed versions of moduli spaces of quiver representations. This factorization formula is related to wall-crossing formulas for Donaldson-Thomas type invariants of M. Kontsevich and Y. Soibelman [4].
\end{abstract}

\section{Introduction}

In 4, a framework for the definition of Donaldson-Thomas type invariants for Calabi-Yau categories endowed with a stability structure is developed. One of the key features of this setup is a wall-crossing formula for these invariants, describing their behaviour under a change of stability structure in terms of a factorization formula for automorphisms of certain Poisson algebras defined using the Euler form of the category.

In this paper, we study such factorization formulas using quiver representations, their moduli spaces, and Hall algebras (for different such approaches, see [1, 3]).

With a quiver without oriented cycles, we associate a Poisson structure on a formal power series ring and study factorizations of a certain automorphism into infinite ordered products associated with stabilities for the quiver (see section 2 for precise definitions). Our main result, Theorem 2.1. describes these factorizations in terms of generating series for Euler characteristics of framed versions of moduli spaces of representations of the quiver, called smooth models in 2]. This result yields a weak form of an integrality conjecture for DonaldsonThomas type invariants of [4].

We approach this theorem by producing the desired factorizations in the Hall algebra of the quiver, in terms of the Harder-Narasimhan recursion of [6]; section 4 therefore adapts some of the material of [6] to the present setup. Using 
an evaluation map, simple identities in the Hall algebra often lead to interesting identities in a skew formal power series ring associated with the quiver (see [7] for some instances of this principle). In the present setup, the latter ring is viewed as a quantization of the Poisson algebra; we develop a simple algebraic setup for constructing automorphisms of the Poisson algebra from a class of formal series in section 3. The key feature is that certain series in the skew formal power series ring induce non-trivial automorphisms of the Poisson algebra via conjugation. That this property applies to the generating series induced from the Harder-Narasimhan recursion follows from a formula for the Betti numbers of smooth models of [2]; this is explained in section 5. We end with two classes of examples in section 6. considering generalized Kronecker quivers, we can prove a weak form of the integrality conjecture of [4. We also relate the factorization formula Theorem 2.1 to a factorization formula of [3] in terms of Gromov-Witten theory. Finally, we consider Dynkin quivers, for which the relevant moduli spaces are trivial.

Acknowledgments: I would like to thank J. Alev, T. Bridgeland, B. Keller, S. Mozgovoy, R. Pandharipande, B. Siebert, Y. Soibelman and V. ToledanoLaredo for interesting discussions concerning the material in this paper. I am indebted to Y. Soibelman for providing me with a preliminary version of [4], and for pointing out a gap in an earlier version of this paper.

\section{Statement of the result}

Let $Q$ be a finite quiver, given by a finite set of vertices $I$ and a finite set of arrows, written as $\alpha: i \rightarrow j$ for $i, j \in I$. We assume throughout the paper that $Q$ has no oriented cycles, thus we can order the vertices as $I=\left\{i_{1}, \ldots, i_{r}\right\}$ in such a way that $k>l$ provided there exists an arrow $i_{k} \rightarrow i_{l}$. Set $\Lambda=\mathbf{Z} I$ with natural basis $i \in I$, and consider the sublattice $\Lambda^{+}=\mathbf{N} I$. Let $\Theta \in \Lambda^{*}$ be a functional (called a stability), and define the slope of a non-zero $d=\sum_{i \in I} d_{i} i \in \Lambda^{+}$as

$$
\mu(d)=\frac{\Theta(d)}{\operatorname{dim} d},
$$

where $\operatorname{dim} d=\sum_{i \in I} d_{i}$. For $\mu \in \mathbf{Q}$, define $\Lambda_{\mu}^{+}$as the subsemigroup of $\Lambda^{+}$of all $0 \neq d \in \Lambda^{+}$such that $\mu(d)=\mu$, together with $0 \in \Lambda$.

Define a bilinear form $\langle$,$\rangle , the Euler form, on \Lambda$ via

$$
\langle d, e\rangle=\sum_{i \in I} d_{i} e_{i}-\sum_{\alpha: i \rightarrow j} d_{i} e_{j} \text { for } d, e \in \Lambda .
$$

Denote by $\{$,$\} the skew-symmetrization of \langle$,$\rangle , thus \{d, e\}=\langle d, e\rangle-\langle e, d\rangle$. Define $b_{i j}=\{i, j\}$ for $i, j \in I$.

We consider the formal power series ring $B=\mathbf{Q}\left[\left[\Lambda^{+}\right]\right]=\mathbf{Q}\left[\left[x_{i}: i \in I\right]\right]$ with 
topological basis $x^{d}=\prod_{i \in I} x_{i}^{d_{i}}$ for $d \in \Lambda^{+}$. The algebra $B$ becomes a Poisson algebra via the Poisson bracket

$$
\left\{x_{i}, x_{j}\right\}=b_{i j} x_{i} x_{j} \text { for } i, j \in I .
$$

Define automorphisms $T_{d}$ of $B$ by

$$
T_{d}\left(x_{j}\right)=x_{j} \cdot\left(1+x^{d}\right)^{\{d, j\}}
$$

for all $d \in \Lambda^{+}$and $j \in I$. A direct calculation shows that these are indeed Poisson automorphisms of $B$ (see Lemma 3.5 for a conceptual proof).

We study a factorization property in the group $\operatorname{Aut}(B)$ of Poisson automorphisms of $B$ involving a descending product $\prod_{\mu \in \mathbf{Q}}^{\leftarrow}$ indexed by rational numbers. In the following sections, we will see that we actually work in a subgroup of $\operatorname{Aut}(B)$ where such products are well-defined a priori (see the remark following Definition 4.2).

Theorem 2.1 In the group Aut $(B)$, we have a factorization

$$
T_{i_{1}} \circ \ldots \circ T_{i_{r}}=\prod_{\mu \in \mathbf{Q}}^{\leftarrow} T_{\mu}
$$

where

$$
T_{\mu}\left(x_{j}\right)=x_{j} \cdot \prod_{i \in I} Q_{\mu}^{i}(x)^{b_{i j}}
$$

for formal series $Q_{\mu}^{i}(x) \in \mathbf{Z}\left[\left[\Lambda_{\mu}^{+}\right]\right]$. These series $Q_{\mu}^{i}(x)$ are given by the generating function

$$
Q_{\mu}^{i}(x)=\sum_{d \in \Lambda_{\mu}^{+}} \chi\left(M_{d, i}^{\Theta}(Q)\right) x^{d},
$$

where $\chi\left(M_{d, i}^{\Theta}(Q)\right)$ denotes the Euler characteristic in singular cohomology of a framed moduli space of semistable representations of $Q$ of dimension vector d (see section 5.1 for the precise definition).

\section{Quantization}

In this section, we quantize the Poisson algebra $B$ to a skew formal power series ring $A$. We define a class of invertible elements of $A$ which induce well-defined non-trival automorphisms of $B$ via conjugation.

Definition 3.1 For a commutative ring $S$ and an invertible element $q \in S^{*}$, define a skew formal power series ring $S_{q}\left[\left[\Lambda^{+}\right]\right]$as follows: as an $S$-module, $S_{q}\left[\left[\Lambda^{+}\right]\right]$has a topological basis consisting of elements $t^{d}$ for $d \in \Lambda^{+}$; multiplication is defined by

$$
t^{d} \cdot t^{e}=q^{-\langle e, d\rangle} t^{d+e} .
$$


We apply this definition to the base $\operatorname{ring} K=\mathbf{Q}(q)$, the field of rational functions in $q$, and to $R=\mathbf{Z}\left[q, q^{-1}\right]$. This yields $A=\mathbf{Q}(q)_{q}\left[\left[\Lambda^{+}\right]\right]$, with a natural $R$ sublattice $\mathcal{A}=R_{q}\left[\left[\Lambda^{+}\right]\right]$.

Lemma 3.2 Specialization at $q=1$ identifies $B$ with $\mathcal{A} /(q-1) \mathcal{A}$ as a Poisson algebra.

Proof: That specialization at $q=1$ induces an isomorphism of $\mathbf{Q}$-algebra follows from the definitions. Moreover, we have

$$
\left\{t^{d}, t^{e}\right\}=\frac{t^{d} t^{e}-t^{e} t^{d}}{q-1}=\frac{q^{-\langle e, d\rangle}-q^{-\langle d, e\rangle}}{q-1} t^{d+e}=\{d, e\} t^{d+e} \bmod (q-1) .
$$

For a series $P(t)=\sum_{d} a_{d}(q) t^{d} \in \mathcal{A}$, we denote by $\bar{P}(x)$ its specialization at $q=1$ in $B$, that is,

$$
\bar{P}(x)=\sum_{d} a_{d}(1) x^{d} .
$$

Any $R$-algebra automorphism of $\mathcal{A}$ induces a Poisson automorphism of $B$ via specialization; we define a class of automorphisms given by conjugation with invertible elements of $A$ mapping $\mathcal{A}$ to itself.

For a functional $\eta \in \Lambda^{*}$, define a twisted form $P\left(q^{\eta} t\right) \in A$ of $P(t)$ by

$$
P\left(q^{\eta} t\right)=\sum_{d \in \Lambda^{+}} q^{\eta(d)} a_{d} t^{d}
$$

We view any $n \in \Lambda$ as a functional $n \cdot \in \Lambda^{*}$ by $n \cdot d=\sum_{i \in I} n_{i} d_{i}$. A series $P(t) \in A$ with $P(0)=1$, i.e. with constant term equal to 1 , is invertible in $A$.

Proposition 3.3 Let $P(t) \in A$ be a series with constant term equal to 1 , and define $Q^{\eta}(t)=P\left(q^{\eta} t\right) P(t)^{-1}$ for all $\eta \in \Lambda^{*}$.

1. The following conditions on $P(t)$ are equivalent:

(a) $Q^{\eta} \in \mathcal{A}$ for all $\eta \in \Lambda^{*}$,

(b) $Q^{i \cdot} \in \mathcal{A}$ for all $i \in I$.

2. Conjugation by $P(t)$ maps $t^{d}$ to $t^{d} \cdot Q^{\{-, d\}}(t)$ for all $d \in \Lambda^{+}$.

3. If the conditions of part 1 are fulfilled, conjugation by $P(t)$ induces the following Poisson automorphism of B:

$$
x^{d} \mapsto x^{d} \cdot \overline{Q^{\{-, d\}}}(x) .
$$

4. We have $\overline{Q^{\eta}}(x)=\prod_{i \in I} \overline{Q^{i} \cdot}(x)^{\eta(i)}$ for all $\eta \in \Lambda^{*}$.

5. The set of all $P(t) \in A$ with $P(0)=1$ fulfilling the conditions of part 1 forms a subgroup $\mathcal{S}$ of $A^{*}$. 
Proof: We have

$Q^{\eta+\nu}(t)=P\left(q^{\eta+\nu} t\right) P(t)^{-1}=P\left(q^{\eta+\nu} t\right) P\left(q^{\nu} t\right)^{-1} P\left(q^{\nu} t\right) P(t)^{-1}=Q^{\eta}\left(q^{\nu} t\right) Q^{\nu}(t)$.

We have $Q^{0}(t)=1$ by the definitions, so $1=Q^{\eta+(-\eta)}(t)=Q^{\eta}\left(q^{-\eta} t\right) Q^{-\eta}(t)$ and thus

$$
Q^{-\eta}(t)=Q^{\eta}\left(q^{-\eta} t\right)^{-1}
$$

We conclude that every $Q^{\eta}(t)$ can be expressed as a product of twisted forms of the $Q^{i \cdot}(t)$ for $i \in I$, proving part 1 and part 4 .

We have $t^{e} t^{d}=q^{\{e, d\}} t^{d} t^{e}$ by definition of $A$; it follows that

$$
P(t) t^{d}=\sum_{e} a_{e}(q) t^{e} t^{d}=t^{d} \sum_{e} q^{\{e, d\}} a_{e}(q) t^{e}=t^{d} P\left(q^{\{-, d\}} t\right),
$$

thus conjugation by $P(t)$ maps $t^{d}$ to $t^{d} P\left(q^{\{-, d\}} t\right) P(t)^{-1}$, proving part 2, Now part 3 follows.

To prove that $\mathcal{S}$ is a subgroup, suppose we are given $P_{1}(t), P_{2}(t) \in \mathcal{S}$, with which we associate elements $Q_{1}^{\eta}(t), Q_{2}^{\eta}(t) \in \mathcal{A}$ as above. We write $Q_{2}^{\eta}(t)=\sum_{e} b_{e}(q) t^{e}$. Then

$$
\begin{aligned}
P_{1}\left(q^{\eta} t\right) P_{2}\left(q^{\eta} t\right) P_{2}(t)^{-1} P_{1}(t)^{-1} & =P_{1}\left(q^{\eta} t\right) Q_{2}^{\eta}(t) P_{1}(t)^{-1} \\
& =P_{1}\left(q^{\eta} t\right) \sum_{e} b_{e}(q) t^{e} P_{1}(t)^{-1} \\
& =\sum_{e} b_{e}(q) t^{e} P_{1}\left(q^{\eta+\{-, e\}} t\right) P_{1}(t)^{-1} \\
& =\sum_{e} b_{e}(q) t^{e} Q_{1}^{\eta+\{-, e\}}(t)
\end{aligned}
$$

by the identities above. Now every summand in the last sum has coefficients in $R$, thus the sum belongs to $\mathcal{A}$. For $P \in \mathcal{S}$, write $P\left(q^{\eta} t\right)^{-1} P(t)=\sum_{d} c_{d}(q) t^{d}$. We conjugate this equation by $P(t)$ and use part 2

$$
Q^{\eta}(t)^{-1}=P(t) P\left(q^{\eta} t\right)^{-1}=P(t) P\left(q^{\eta} t\right)^{-1} P(t) P(t)^{-1}=\sum_{d} c_{d}(q) t^{d} \underbrace{Q^{\{-, d\}}(t)}_{\in \mathcal{A}} .
$$

The leftmost term belonging to $\mathcal{A}$, we see inductively that all $c_{d}(q)$ belong to $R$. This proves part 5 .

Corollary 3.4 With notation of the previous proposition, the map sending $P(t) \in \mathcal{S}$ to the automorphism

$$
x^{d} \mapsto x^{d} \cdot \overline{Q^{\{-, d\}}}(x)
$$

defines a group homomorphism $\Phi: \mathcal{S} \rightarrow \operatorname{Aut}(B)$. 
As a first example of series in $\mathcal{S}$, we choose a dimension vector $d \in \Lambda^{+}$such that $\langle d, d\rangle=1$ (a real root for the root system associated with $Q$ ), and consider the series

$$
P_{d}(t)=\sum_{n=0}^{\infty} \frac{q^{-n^{2}}}{\left(1-q^{-1}\right) \cdot \ldots \cdot\left(1-q^{-n}\right)} t^{n d} \in A .
$$

The following can be proved using standard identities involving the $q$-binomial coefficients $\left[\begin{array}{c}M \\ N\end{array}\right]=\frac{\left(q^{M}-1\right) \cdot \ldots \cdot\left(q^{M-N+1}-1\right)}{\left(q^{N}-1\right) \cdot \ldots \cdot(q-1)}$. We will give a more conceptual proof in Remark 5.2 .

Lemma 3.5 For any $\eta \in \Lambda$, we have

$$
Q_{d}^{\eta}(t)=P_{d}\left(q^{\eta} t\right) P_{d}(t)^{-1}=\sum_{n=0}^{\infty}\left[\begin{array}{c}
\eta(d) \\
n
\end{array}\right] t^{n d} .
$$

Using Corollary 3.4, we get in the notation of section 2.

$$
\Phi\left(P_{d}(t)\right)=T_{d} \in \operatorname{Aut}(B) .
$$

For later reference, we note the following property:

Lemma 3.6 Suppose $P(t) \in \mathcal{S}$ belongs to $\mathbf{Q}(q)_{q}\left[\left[\Lambda_{\mu}^{+}\right]\right]$for some $\mu \in \mathbf{Q}$, and let $Q^{\eta}(t)$ be as in Proposition 3.3. Then $Q^{\eta}(t)$ and $Q^{\nu}(t)$ coincide if $\eta-\nu$ is a rational multiple of the functional $\Theta-\mu \cdot \operatorname{dim}$.

Proof: The subsemigroup $\Lambda_{\mu}^{+}$has the defining condition $\mu(d)=\mu$, that is, $(\Theta-\mu \cdot \operatorname{dim})(d)=0$. Thus, the condition in the statement of the lemma is equivalent to $\eta(d)=\nu(d)$ for all $d \in \Lambda_{\mu}^{+}$. In the definition of $Q^{\eta}(t)$, the functional $\eta$ only enters through its values on $\Lambda_{\mu}^{+}$by the choice of $P(t)$; the lemma follows.

\section{Hall algebras and the Harder-Narasimhan re- cursion}

With a quiver $Q$ and a stability $\Theta$ as before, we associate a system of rational functions defined recursively, and relate it to the cohomology of quiver moduli via Hall algebras; we adapt material of [6] to the present setup. Generating series for this system of rational functions will yield the automorphisms $T_{\mu}$ of Theorem 2.1 via the map $\Phi$ of Corollary 3.4 .

\subsection{The Harder-Narasimhan recursion}

Definition 4.1 Define the following rational functions and their generating series: 
1. for $d \in \Lambda^{+}$, define $e_{d}(q) \in \mathbf{Q}(q)$ by

$$
e_{d}(q)=q^{-\langle d, d\rangle} \prod_{i \in I} \prod_{j=1}^{d_{i}}\left(1-q^{-j}\right)^{-1} .
$$

2. Define $p_{d}(q) \in \mathbf{Q}(q)$ for $d \in \Lambda^{+}$recursively as follows: if $\Theta$ is constant on $\operatorname{supp}(d)=\left\{i \in I: d_{i} \neq 0\right\}$, then $p_{d}(q)=e_{d}(q)$. Otherwise, define

$$
p_{d}(q)=e_{d}(q)-\sum_{d^{*}} q^{-\sum_{k<l}\left\langle d^{l}, d^{k}\right\rangle} p_{d^{1}}(q) \cdot \ldots \cdot p_{d^{s}}(q),
$$

where the sum runs over all non-trivial decompositions $d=d^{1}+\ldots+d^{s}$ (i.e. $s \geq 2$ and $d^{k} \neq 0$ for all $k$ ) such that $\mu\left(d^{1}\right)>\ldots>\mu\left(d^{s}\right)$.

3. Define $P(t)=\sum_{d \in \Lambda^{+}} e_{d}(q) t^{d} \in A$ and $P_{\mu}(t)=\sum_{d \in \Lambda_{\mu}^{+}} p_{d}(q) t^{d} \in A$ for all $\mu \in \mathbf{Q}$.

Remark: The following explicit formula for $p_{d}(q)$ (a resolution of the defining recursion) is proved in [6]:

$$
p_{d}(q)=\sum_{d^{*}}(-1)^{s-1} q^{-\sum_{k \leq l}\left\langle d^{l}, d^{k}\right\rangle} \prod_{k=1}^{s} \prod_{i \in I} \prod_{j=1}^{d_{i}}\left(1-q^{-j}\right)^{-1} \in \mathbf{Q}(q),
$$

where the sum ranges over all tuples $d^{*}=\left(d^{1}, \ldots, d^{s}\right)$ of non-zero dimension vectors such that $d=d^{1}+\ldots+d^{s}$ and $\mu\left(d^{1}+\ldots+d^{k}\right)>\mu(d)$ for all $k<s$.

Definition 4.2 Given elements $c_{\mu} \in \mathbf{Q}(q)_{q}\left[\left[\Lambda_{\mu}^{+}\right]\right]$for $\mu \in \mathbf{Q}$ such that $c_{\mu}(0)=$ 0 , we define

$$
\prod_{\mu \in \mathbf{Q}}^{\leftarrow}\left(1+c_{\mu}\right)=\sum_{\mu_{1}>\ldots>\mu_{s}} c_{\mu_{1}} \cdot \ldots \cdot c_{\mu_{s}}
$$

Remark: The sum on the right hand side is indeed well-defined, since calculation of each $t^{d}$-coefficient reduces to a finite sum. Applying this definition to series $R_{\mu} \in \mathcal{S} \cap \mathbf{Q}(q)_{q}\left[\left[\Lambda_{\mu}^{+}\right]\right]$, we see that decreasing products $\prod_{\mu \in \mathbf{Q}}^{\leftarrow}$ in the image of $\Phi: \mathcal{S} \rightarrow \operatorname{Aut}(B)$ are well-defined via

$$
\prod_{\mu \in \mathbf{Q}}^{\leftarrow} \Phi\left(R_{\mu}\right)=\Phi\left(\prod_{\mu \in \mathbf{Q}}^{\leftarrow} R_{\mu}\right)
$$

Lemma 4.3 We have $P_{i_{1}}(t) \cdot \ldots \cdot P_{i_{r}}(t)=P(t)=\prod_{\mu \in \mathbf{Q}}^{\leftarrow} P_{\mu}(t)$ in $A$.

Proof: We first prove the second identity. By the definition of the functions $p_{d}(q)$, we have

$$
\prod_{\mu \in \mathbf{Q}}^{\leftarrow} P_{\mu}(t)=\sum_{\mu_{1}>\ldots>\mu_{s}}\left(\sum_{d \in \Lambda_{\mu_{1}}^{+} \backslash 0} p_{d}(q) t^{d}\right) \cdot \ldots \cdot\left(\sum_{d \in \Lambda_{\mu_{s}}^{+} \backslash 0} p_{d}(q) t^{d}\right)
$$




$$
\begin{aligned}
& =\sum_{\substack{\left(d^{1}, \ldots, d^{s}\right) \\
\mu\left(d^{1}\right)>\ldots>\mu\left(d^{s}\right)}} p_{d^{1}}(q) t^{d^{1}} \cdot \ldots \cdot p_{d^{s}}(q) t^{d^{s}} \\
& =\sum_{\substack{\left(d^{1}, \ldots, d^{s}\right) \\
\mu\left(d^{1}\right)>\ldots>\mu\left(d^{s}\right)}} q^{-\sum_{k<l}\left\langle d^{l}, d^{k}\right\rangle} p_{d^{1}}(q) \cdot \ldots \cdot p_{d^{s}}(q) t^{d^{1}+\ldots+d^{s}} \\
& =\sum_{d} e_{d}(q) t^{d}=P_{d}(t) .
\end{aligned}
$$

The first identity follows from the definition of $A$ and the choice of the ordering of the vertices of $Q$; we have

$$
\begin{aligned}
P_{i_{1}}(t) \cdot \ldots \cdot P_{i_{r}}(t) & =\sum_{d_{1}, \ldots, d_{r} \geq 0} q^{-\sum_{i \in I} d_{i}^{2}} \prod_{i \in I} \prod_{j=1}^{d_{i}}\left(1-q^{-j}\right)^{-1} t^{d_{1} i_{1}} \cdot \ldots \cdot t^{d_{r} i_{r}} \\
& =\sum_{d \in \Lambda^{+}} q^{\sum_{\alpha: i \rightarrow j} d_{i} d_{j}-\sum_{i} d_{i}^{2}} \prod_{i \in I} \prod_{j=1}^{d_{i}}\left(1-q^{-j}\right)^{-1} t^{d}=P(t) .
\end{aligned}
$$

\subsection{Quiver representations}

Let $k$ be a field. We consider finite-dimensional $k$-representations

$$
M=\left(\left(M_{i}\right)_{i \in I},\left(M_{\alpha}: M_{i} \rightarrow M_{j}\right)_{\alpha: i \rightarrow j}\right)
$$

of $Q$, given by an $I$-tuple of finite-dimensional $k$-vector spaces $M_{i}$, together with $k$-linear maps $M_{\alpha}: M_{i} \rightarrow M_{j}$ indexed by the arrows $\alpha: i \rightarrow j$. Let $\bmod _{k} Q$ be the abelian $k$-linear category of finite dimensional $k$-representations of $Q$. The Grothendieck group of $\bmod _{k} Q$ can be identified with the lattice $\Lambda$ via the map attaching to the representation $M$ its dimension vector

$$
\underline{\operatorname{dim}} M=\sum_{i}\left(\operatorname{dim}_{k} M_{i}\right) i
$$

The above bilinear form $\left\langle_{-,}\right\rangle$then becomes the homological Euler form on $\bmod _{k} Q$, in the sense that

$$
\langle\underline{\operatorname{dim}} M, \underline{\operatorname{dim}} N\rangle=\operatorname{dim} \operatorname{Hom}_{Q}(M, N)-\operatorname{dim} \operatorname{Ext}_{Q}^{1}(M, N)
$$

for all representations $M, N$.

We denote by $S_{i}$ the one-dimensional simple representation supported at the vertex $i \in I$, and by $P_{i}$ its projective cover. Then every projective representation of $Q$ is isomorphic to $P^{(n)}=\bigoplus_{i \in I} P_{i}^{n_{i}}$ for some $n \in \Lambda^{+}$.

For the following basic notions and facts on (semi-)stability of quiver representations, see e.g. [7. For a non-zero representation $M$, we define its slope as the 
slope of its dimension vector, i.e. $\mu(M)=\mu(\underline{\operatorname{dim}} M)$. We call $M$ semistable if $\mu(U) \leq \mu(M)$ for all non-zero proper subrepresentations $U$ of $M$, and we call $M$ stable if $\mu(U)<\mu(M)$ for all such $U$. Moreover, we call $M$ polystable if it is isomorphic to a direct sum of stable representations of the same slope.

The semistable representations of a fixed slope $\mu \in \mathbf{Q}$ form a full abelian subcategory $\bmod _{k}^{\mu} Q$, whose simple (resp. semisimple) objects are given by the stable (resp. polystable) representations of slope $\mu$.

For every representation $M$, there exists a unique Harder-Narasimhan filtration, by which we mean a filtration

$$
0=M_{0} \subset M_{1} \subset \ldots \subset M_{s}=M
$$

such that all subfactors $M_{i} / M_{i-1}$ are semistable, and

$$
\mu\left(M_{1} / M_{0}\right)>\ldots>\mu\left(M_{s} / M_{s-1}\right) .
$$

\subsection{Hall algebras}

Let $k$ be a finite field. For $d \in \Lambda^{+}$, fix $k$-vector spaces $M_{i}$ of dimension $d_{i}$ for $i \in I$, and let

$$
R_{d}=\bigoplus_{\alpha: i \rightarrow j} \operatorname{Hom}_{k}\left(M_{i}, M_{j}\right)
$$

be the space of all $k$-representations of $Q$ on the vector spaces $M_{i}$, on which the group

$$
G_{d}=\prod_{i \in I} \mathrm{GL}\left(M_{i}\right)
$$

acts via base change

$$
\left(g_{i}\right)_{i} \cdot\left(M_{\alpha}\right)_{\alpha}=\left(g_{j} M_{\alpha} g_{i}^{-1}\right)_{\alpha: i \rightarrow j},
$$

such that the $G_{d^{-}}$orbits in $R_{d}$ correspond naturally to the isomorphism classes of representations of $Q$. Let $\mathbf{Q}^{G_{d}}\left(R_{d}\right)$ be the space of (arbitrary) $G_{d}$-invariant $\mathrm{Q}$-valued functions on $R_{d}$, and define

$$
H_{k}((Q))=\prod_{d \in \Lambda^{+}} \mathbf{Q}^{G_{d}}\left(R_{d}\right)
$$

This becomes a $\Lambda^{+}$-graded algebra, the (completed) Hall algebra of $Q$, via the convolution type product

$$
(f g)(M)=\sum_{U \subset M} f(U) g(M / U)
$$

for functions $f \in \mathbf{Q}^{G_{d}}\left(R_{d}\right), g \in \mathbf{Q}^{G_{e}}\left(R_{e}\right)$ and representations $M \in R_{d+e}$. Note that the sum over all subrepresentations $U$ is finite and that the value of $f$ on $U$ (resp. of $g$ on $M / U$ ) is well-defined by the definitions. 
Let $\mathbf{Q}_{|k|}\left[\left[\Lambda^{+}\right]\right]$be defined as in Definition [3.1, where the cardinality $|k|$ of $k$ is viewed as an element of $\mathbf{Q}$. The map

$$
\int: H((Q)) \rightarrow \mathbf{Q}_{|k|}\left[\left[\Lambda^{+}\right]\right]
$$

given by

$$
\int f=\frac{1}{\left|G_{d}\right|} \sum_{M \in R_{d}} f(M) t^{d}
$$

for $f \in \mathbf{Q}^{G_{d}}\left(R_{d}\right)$ is a $\mathbf{Q}$-algebra morphism by $[\underline{6}$.

Define $1_{d}=1_{R_{d}}$ as the characteristic function of $R_{d}$, and define $1_{d}^{s s t}=1_{R_{d}^{s s t}}$ as the characteristic function of the locus $R_{d}^{s s t}$ of semistable representations in $R_{d}$. We form generating functions of these elements by

$$
e:=\sum_{d \in \Lambda^{+}} 1_{d}, \quad e_{\mu}=\sum_{d \in \Lambda_{\mu}^{+}} 1_{d}^{s s t} \text { for } \mu \in \mathbf{Q} .
$$

Lemma 4.4 For every $d \in \Lambda^{+}$, we have the following identity in $H_{k}((Q))$ :

$$
1_{d}=\sum_{d^{*}} 1_{d^{1}}^{s s t} \cdot \ldots \cdot 1_{d^{s}}^{s s t}
$$

the sum running over all decompositions $d=d^{1}+\ldots+d^{s}$ into non-zero dimension vectors such that $\mu\left(d^{1}\right)>\ldots>\mu\left(d^{s}\right)$. Consequently, we have $e=\prod_{\mu \in \mathbf{Q}}^{\leftarrow} e_{\mu}$ in $H_{k}((Q))$.

Proof: The existence and uniqueness of the Harder-Narasimhan filtration (see section 4.2) can be rephrased as follows using the definition of the Hall algebra: for every $k$-representation $M$ of $Q$, there exists a unique tuple $\left(d^{1}, \ldots, d^{s}\right)$ of dimension vectors such that $\mu\left(d^{1}\right)>\ldots>\mu\left(d^{s}\right)$ and

$$
\left(1_{d^{1}}^{s s t} \cdot \ldots \cdot 1_{d^{s}}^{s s t}\right)(M)=1
$$

(namely, the $d^{i}$ are the dimension vectors of the subfactors in the HarderNarasimhan filtration). The first identity follows. Similar to the proof of Lemma 4.3 above, this allows us to compute

$$
\begin{aligned}
\prod_{\mu \in \mathbf{Q}}^{\leftarrow} e_{\mu} & =\sum_{\mu_{1}>\ldots>\mu_{s}}\left(\sum_{d \in \Lambda_{\mu_{1}}^{+} \backslash 0} 1_{d}^{s s t}\right) \cdot \ldots \cdot\left(\sum_{d \in \Lambda_{\mu_{s}}^{+} \backslash 0} 1_{d}^{s s t}\right) \\
& =\sum_{\substack{\left(d^{1}, \ldots ., d^{s}\right) \\
\mu\left(d^{1}\right)>\ldots>\mu\left(d^{s}\right)}} 1_{d^{1}}^{s s t} \cdot \ldots \cdot 1_{d^{s}}^{s s t} \\
& =\sum_{d \in \Lambda^{+}} 1_{d}=e_{d}
\end{aligned}
$$

Since the rational functions $e_{d}(q)$ and $p_{d}(q)$ have no poles at $q=|k|$ by Definition 4.1, we can specialize the generating functions $P(t)$ and $P_{\mu}(t)$ to $\mathbf{Q}_{|k|}\left[\left[\Lambda^{+}\right]\right]$. 
Proposition 4.5 The series $P(t)$ specializes to $\int e$, and the series $P_{\mu}(t)$ specialize to $\int e_{\mu}$ for all $\mu \in \mathbf{Q}$.

Proof: By the definitions of $R_{d}, G_{d}$ and the Euler form $\left\langle_{-,}\right\rangle$on $Q$, we have

$$
\int 1_{d}=\frac{\left|R_{d}\right|}{\left|G_{d}\right|}=\frac{|k|^{\sum_{\alpha: i \rightarrow j} d_{i} d_{j}}}{\prod_{i \in I}\left|\mathrm{GL}_{d_{i}}(k)\right|}=|k|^{-\langle d, d\rangle} \prod_{i \in I} \prod_{j=1}^{d_{i}}\left(1-|k|^{-j}\right)^{-1}=e_{d}(|k|),
$$

proving the first statement. The second now follows since the elements $\int 1_{d}^{\text {sst }}$ satisfy the same recursion as the elements $p_{d}(q)$ by Lemma 4.4 .

Remark: By the definition of the function $1_{d}^{s s t}$, we thus have

$$
p_{d}(|k|)=\frac{\left|R_{d}^{s s t}\right|}{\left|G_{d}\right|} .
$$

In case $d$ is coprime for $\Theta$, this is used in [6] to prove that

$$
(q-1) \cdot p_{d}(q)=\sum_{i} \operatorname{dim} H^{i}\left(M_{d}^{s t}(Q), \mathbf{Q}\right) q^{i / 2},
$$

where $M_{d}^{s t}(Q)$ denotes the moduli space of stable representations of $Q$ of dimension vector $d$ (see section 5.1 for the definitions). The result holds since in the coprime case, we have a smooth projective moduli space whose numbers of rational points over finite fields $k$ behave polynomially in $|k|$.

\section{Smooth models and the proof of Theorem 2.1}

Using the construction of (framed versions of) moduli spaces of representations of quivers, we prove that the series $P_{\mu}$ belong to $\mathcal{S}$. This fact, together with the Harder-Narasimhan recursion of the previous section, proves Theorem 2.1 .

\subsection{Quiver moduli}

In this section, we work over the complex numbers. For every dimension vector $d \in \Lambda^{+}$, there exists a smooth complex variety $M_{d}^{s t}(Q)$ whose points parametrize isomorphim classes of stable complex representations of $Q$ of dimension vector $d$. It embeds as an open subset into a (typically singular) projective variety $M_{d}^{s s t}(Q)$ whose points parametrize isomorphism classes of polystable representations of $Q$ of dimension vector $d$. If $d$ is coprime for $\Theta$, by which we mean that $\mu(e) \neq \mu(d)$ for all $0 \leq e<d$, we have $M_{d}^{s t}(Q)=M_{d}^{s s t}(Q)$, consequently a smooth projective complex variety.

For $n \in \Lambda^{+}$, fix additional vector spaces $V_{i}$ of dimension $n_{i}$ for $i \in I$.

Theorem 5.1 ([2]) There exists a projective variety $M_{d, n}(Q)$ parametrizing equivalence classes of pairs $(M, f)$ consisting of a semistable representation $M$ of 
$Q$ on the vector spaces $M_{i}$, together with a tuple of maps $f=\left(f_{i}: V_{i} \rightarrow M_{i}\right)_{i \in I}$ such that $\mu(U)<\mu(M)$ whenever $U \subset M$ is a proper subrepresentation of $M$ containing the image of $f$ (that is, the subrepresentation generated by the $f_{i}\left(V_{i}\right)$ ); such pairs are considered up to isomorphisms of the representations intertwining the additional maps, that is, $(M, f)$ is equivalent to $\left(M^{\prime}, f^{\prime}\right)$ if there exists an isomorphism $\varphi: M \rightarrow M^{\prime}$ such that $f^{\prime}=\varphi f$.

This is a framed version of moduli of (semistable) quiver representations, called smooth models in [2]. There it is also shown that the framing datum $f$ induces a morphism from the projective representation $P^{(n)}$ to $M$ such that $\mu(U)<\mu(M)$ for any proper subrepresentation $U$ of $M$ containing its image.

There exists a canonical projective morphism $\pi: M_{d, n}^{\Theta}(Q) \rightarrow M_{d}^{s s t}(Q)$, which by [2] is étale locally trivial for a suitable (Luna type) stratification of $M_{d}^{s s t}(Q)$ with known fibres (they are isomorphic to certain nilpotent parts of smooth models for the trivial stability and quivers with oriented cycles).

As a special case, we consider the quiver $Q^{0}$ consisting of a single vertex and no arrows, with trivial stability. From the definitions, it is easy to see that $M_{d, n}^{0}\left(Q^{0}\right) \simeq \operatorname{Gr}_{d}^{n}(\mathbf{C})$, the Grassmannian of $k$-planes in $n$-space.

\subsection{Proof of Theorem 2.1}

Let again $k$ be a finite field. The semistable representations of $Q$ of fixed slope

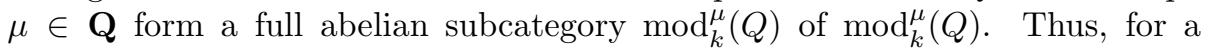
subrepresentation $U \subset M$ of a representation $M \in \bmod _{k}^{\mu}(Q)$, we can form the intersection

$$
\langle U\rangle_{\mu}=\bigcap_{\substack{V \in \bmod _{k}^{\mu}(Q) \\ U \subset V \subset M}} V \in \bmod _{k}^{\mu}(Q) .
$$

This is the minimal subrepresentation of $M$ containing $U$ which is semistable of slope $\mu$. For $n \in \Lambda^{+}$, we denote by $\operatorname{Hom}_{Q}^{0}\left(P^{(n)}, M\right)$ the set of all morphisms $f \in \operatorname{Hom}_{Q}\left(P^{(n)}, M\right)$ such that $\langle\operatorname{Im}(f)\rangle_{\mu}=M$. As mentioned above, the data $(M, f)$ of Theorem 5.1 can be viewed as pairs consisting of a representation $M \in \bmod _{k}^{\mu}(Q)$, together with a map $f \in \operatorname{Hom}_{Q}^{0}\left(P^{(n)}, M\right)$.

For $d \in \Lambda_{\mu}^{+}$, define $1_{d, n}^{s s t}$ as the function taking value $\left|\operatorname{Hom}_{Q}\left(P^{(n)}, M\right)\right|=q^{n \cdot d}$ on $M \in R_{d}^{s s t}$, and value 0 outside $R_{d}^{s s t}$. Define $f_{d, n} \in H_{k}((Q))$ as the function taking value $\left|\operatorname{Hom}_{Q}^{0}\left(P^{(n)}, M\right)\right|$ on $M \in R_{d}^{s s t}$, and value 0 outside $R_{d}^{s s t}$. We form generating functions $e_{\mu, n}=\sum_{d \in \Lambda_{\mu}^{+}} 1_{d, n}^{s s t}$ and $h_{\mu, n}=\sum_{d \in \Lambda_{\mu}^{+}} f_{d, n}$.

The following lemma is a special case of [2, Lemma 5.1]:

Lemma 5.2 We have $e_{\mu, n}=h_{\mu, n} \cdot e_{\mu}$ in $H_{k}((Q))$.

Proof: For a representation $M \in \bmod _{k}^{\mu}(Q)$, we have by the definitions

$$
\left(h_{\mu, n} \cdot e_{\mu}\right)(M)=\sum_{U}\left|\operatorname{Hom}^{0}\left(P^{(n)}, U\right)\right|,
$$


where the sum runs over all subrepresentations $U$ of $M$ such that $U \in \bmod _{k}^{\mu}(Q)$. But the set of all pairs $(U, f)$ consisting of a subrepresentation $U \subset M$ such that $U \in \bmod _{k}^{\mu}(Q)$ and a morphism $f \in \operatorname{Hom}^{0}\left(P^{(n)}, U\right)$ is naturally in bijection to $\operatorname{Hom}\left(P^{(n)}, M\right)$, by associating to $f: P^{(n)} \rightarrow M$ the pair $\left(\langle\operatorname{Im}(f)\rangle_{\mu}, f: P^{(n)} \rightarrow\right.$ $\left.\langle\operatorname{Im}(f)\rangle_{\mu}\right)$. Thus,

$$
\left(h_{\mu, n} \cdot e_{\mu}\right)(M)=\left|\operatorname{Hom}\left(P^{(n)}, M\right)\right|=e_{\mu, n}(M),
$$

proving the lemma.

Proposition 5.3 The series $P_{\mu}(t)$ belongs to $\mathcal{S}$. The automorphism $\Phi\left(P_{\mu}(t)\right)$ is given by

$$
x^{d} \mapsto x^{d} \cdot \prod_{i \in I} Q_{\mu}^{i}(x)^{\{i, d\}},
$$

where

$$
Q_{\mu}^{i}(x)=\sum_{d \in \Lambda_{d}^{+}} \chi\left(M_{d, i}^{\Theta}(Q)\right) x^{d} .
$$

Proof: By Proposition 4.5. we know that the series $P_{\mu}(t)$ specializes to $\int e_{\mu}$ under specialization of $q$ to $k$. By definition of $e_{\mu, n}$, the series $P_{\mu}\left(q^{n \cdot} t\right)$ thus specializes to $\int e_{\mu, n}$ for $n \in \Lambda^{+}$. By the previous lemma, the function $Q_{\mu}^{n \cdot}(t)=$ $P_{\mu}\left(q^{n \cdot} t\right) P_{\mu}(t)^{-1}$ thus specializes to $\int e_{\mu, n}^{0}$. By definition, the function $f_{d, n}$ integrates to

$$
\int f_{d, n}=\frac{\mid\left\{(M, f): M \in R_{d}^{s s t}, f \in \operatorname{Hom}^{0}\left(P^{(n)}, M\right\} \mid\right.}{\left|G_{d}\right|} .
$$

As in the proof of 2, Theorem 5.2], we can conclude, using the definition of the smooth model $M_{d, n}^{\Theta}(Q)$ and the comparison between numbers of rational points and Betti numbers as in the final remark of section 4.3, that the Poincare polynomial $\sum_{i} \operatorname{dim} H^{i}\left(M_{d, n}^{\Theta}(Q), \mathbf{Q}\right) q^{i / 2}$ specializes to $\int f_{d, n}$ at $q=|k|$. Consequently, we have

$$
\int e_{\mu, n}=\sum_{d \in \Lambda_{\mu}^{+}} \sum_{i} \operatorname{dim} H^{i}\left(M_{d, n}^{\Theta}(Q), \mathbf{Q}\right) q^{i / 2} t^{d} .
$$

Thus, the coefficients $b_{d}(q)$ of $Q_{\mu}^{n \cdot}(t)$ are rational functions taking integer values at all prime powers $q$, and thus are polynomials in $q$. By part 1 of Proposition 3.3. we conclude that $P_{\mu}(t) \in \mathcal{S}$. Using parts 3 and 4 of the same proposition, we derive the claimed formula for $\Phi\left(P_{\mu}(t)\right)$ since the Poincare polynomial specializes to the Euler characteristic at $q=1$ in absence of odd cohomology.

Remark: Applying this result to the quiver $Q^{0}$, we get Lemma 3.5 in the case $d=i$. The assumption $\langle d, d\rangle=1$ there allows to generalize to such $d$ using the definition of $P_{d}(q)$.

Theorem 2.1 now follows immediately from Lemma 3.5, Lemma 4.3 and Proposition 5.3 


\section{Examples}

\subsection{Generalized Kronecker quivers}

Let $Q=K_{m}$ be the $m$-arrow Kronecker quiver with set of vertices $I=\{i, j\}$ and $m$ arrows from $j$ to $i$. We have $\{a i+b j, c i+d j\}=m \cdot(a d-b c)$ and in particular $b_{i j}=m$. We choose the stability $\Theta=j^{*}$. Writing a dimension vector $d \in \Lambda^{+}$as $d=a i+b j$ for $a, b \in \mathbf{N}$, the automorphism $T_{a, b}=T_{d}$ of the Poisson algebra $B_{m}=\mathbf{Q}\left[\left[x_{i}, x_{j}\right]\right]$ with Poisson bracket $\left\{x_{i}, x_{j}\right\}=m x_{i} x_{j}$ is then given by

$$
T_{a, b}:\left\{\begin{aligned}
x_{i} & \mapsto x_{i}\left(1+x_{i}^{a} x_{j}^{b}\right)^{-m b} \\
x_{j} & \mapsto x_{j}\left(1+x_{i}^{a} x_{j}^{b}\right)^{m a}
\end{aligned}\right\} .
$$

We prove the following weak version of [4, Conjecture 1]:

Theorem 6.1 In $\operatorname{Aut}\left(B_{m}\right)$, there exists a factorization

$$
T_{i} \circ T_{j}=\prod_{b / a \in \mathbf{Q}}^{\leftarrow} T_{a, b}^{d(a, b)},
$$

where $d(a, b) \in \frac{1}{\operatorname{gcd}(a, b)} \mathbf{Z}$ for all $a, b \in \mathbf{N}$.

Proof: By Theorem 2.1, we have a factorization $T_{i} \circ T_{j}=\prod_{\mu \in \mathbf{Q}}^{\leftarrow} T_{\mu}$, where $T_{\mu}$ is given by

$$
T_{\mu}:\left\{\begin{array}{rll}
x_{i} & \mapsto x_{i} \cdot Q_{\mu}^{j}(x)^{-m} \\
x_{j} & \mapsto x_{j} \cdot Q_{\mu}^{i}(x)^{m}
\end{array}\right\}
$$

for $Q_{\mu}^{i}(x), Q_{\mu}^{j}(x)$ being the corresponding generating series for Euler characteristics of smooth models. Writing $\mu=b /(a+b)$ for coprime $a, b \in \mathbf{N}$, we have $\Lambda_{\mu}^{+}=\mathbf{N} \cdot(a, b)$, and thus $Q_{\mu}^{i}(x), Q_{\mu}^{j}(x) \in \mathbf{Z}\left[\left[x_{i}^{a} x_{j}^{b}\right]\right]$. We choose integers $c, d \in \mathbf{Z}$ such that $a c+b d=1$ and define

$$
F_{\mu}(x)=Q_{\mu}^{i}(x)^{c} Q_{\mu}^{j}(x)^{d} \in \mathbf{Z}\left[\left[x_{i}^{a} x_{j}^{b}\right]\right] .
$$

By Lemma 3.6, we have $Q_{\mu}^{j}(x)^{a}=Q_{\mu}^{i}(x)^{b}$. It then follows that

$$
F_{\mu}(x)^{a}=Q_{\mu}^{i}(x) \text { and } F_{\mu}(x)^{b}=Q_{\mu}^{j}(x) .
$$

We can factor $F_{\mu}(x)$ into an infinite product

$$
F_{\mu}(x)=\prod_{k \geq 1}\left(1+\left(x_{i}^{a} x_{j}^{b}\right)^{k}\right)^{c(\mu, k)}
$$

for integers $c(\mu, k) \in \mathbf{Z}$. These two identities allow us to write $T_{\mu}$ in the form

$$
T_{\mu}:\left\{\begin{array}{rll}
x_{i} & \mapsto x_{i} \cdot \prod_{k \geq 1}\left(1+x_{i}^{k a} x_{j}^{k b}\right)^{-m b c(\mu, k)}, \\
x_{j} & \mapsto x_{j} \cdot \prod_{k \geq 1}\left(1+x_{i}^{k a} x_{j}^{k b}\right)^{\operatorname{mac}(\mu, k)}
\end{array}\right\} .
$$


Using the definition of the automorphisms $T_{k a, k b}$ and defining $d(k a, k b)=$ $\frac{1}{k} c(\mu, k)$, the theorem follows.

It is now easy to re-derive the examples of [4, Section 1.4]. For $m=1$, there are three isomorphism classes of stable representations of $K_{1}$, namely the two simple representations $S_{i}, S_{j}$ and their non-trivial extension $X$. Thus, the relevant smooth models reduce to Grassmannians as in the case of the quiver $Q^{0}$, yielding the factorization

$$
T_{1,0} \circ T_{0,1}=T_{0,1} \circ T_{1,1} \circ T_{0,1},
$$

which can also be verified directly.

For $m=2$, we have a unique stable representation for any dimension vector $k i+$ $(k+1) j$ or $(k+1) i+k j$. Moreover, we have a $\mathbf{P}^{1}$-family of stable representations of dimension vector $i+j$, and no stables of dimension vector $k i+k j$ for $k \geq 2$. We have $M_{k i+k j, n}^{\Theta}\left(K_{2}\right) \simeq \operatorname{Hilb}^{k}\left(\mathbf{P}^{1}\right) \simeq \mathbf{P}^{k}$ for $n=i, j$, and thus

$$
Q_{1 / 2}^{i}(x)=Q_{1 / 2}^{j}(x)=\sum_{k \geq 0}(k+1)\left(x_{i} x_{j}\right)^{k}=\left(1-x_{i} x_{j}\right)^{-2} .
$$

We arrive at the following factorization in $B_{2}$ :

$$
T_{1,0} \circ T_{0,1}=\left(T_{0,1} \circ T_{1,2} \circ T_{2,3} \circ \ldots\right) \circ T \circ\left(\ldots \circ T_{3,2} \circ T_{2,1} \circ T_{1,0}\right),
$$

where

$$
T:\left\{\begin{array}{lll}
x_{i} & \mapsto & x_{i} \cdot\left(1-x_{i} x_{j}\right)^{4} \\
x_{j} & \mapsto & x_{j} \cdot\left(1-x_{i} x_{j}\right)^{-4}
\end{array}\right\} .
$$

Remark: In [3, Theorem 0.1], the automorphisms $T_{\mu}$, given by generating functions of Euler characteristics of quiver moduli, are calculated in terms of generating function of genus 0 Gromov-Witten invariants of toric surfaces. This alternative interpretation hints towards a link between the underlying geometries.

\subsection{Dynkin quivers}

Let $Q$ be a quiver of Dynkin type. Then the isomorphism classes of indecomposable representations $U_{\alpha}$ correspond bijectively to the positive roots $\alpha \in \Delta^{+}$ of the corresponding root system. The Harder-Narasimhan filtration of section 4.2 can be replaced by a more explicit filtration (we refer to 5 for details and a related application of this filtration). Namely, the positive roots can be ordered as $\alpha_{1}, \ldots, \alpha_{\nu}$ in such a way that $\operatorname{Hom}_{Q}\left(U_{\alpha_{l}}, U_{\alpha_{k}}\right)=0=\operatorname{Ext}_{Q}^{1}\left(U_{\alpha_{k}}, U_{\alpha_{l}}\right)$ for $k<l$. Now every representation $M$ of $Q$ is of the form $M=\bigoplus_{k} U_{\alpha_{k}}^{m_{k}}$, and thus admits a unique filtration

$$
0=M^{\nu} \subset M^{\nu-1} \subset \ldots \subset M^{1} \subset M^{0}=M
$$


such that $M^{k-1} / M^{k} \simeq U_{\alpha_{k}}^{m_{k}}$ for all $k=1, \ldots, \nu$. The arguments of section 4 can be applied to this filtration, yielding a factorization $P_{i_{1}} \cdot \ldots \cdot P_{i_{r}}=P_{\alpha_{\nu}} \cdot \ldots \cdot P_{\alpha_{1}}$. Applying the map $\Phi: \mathcal{S} \rightarrow \operatorname{Aut}(B)$, we conclude

$$
T_{i_{1}} \circ \ldots \circ T_{i_{r}}=T_{\alpha_{\nu}} \circ \ldots \circ T_{\alpha_{1}} .
$$

\section{References}

[1] T. Bridgeland, V. Toledano-Laredo: Stability conditions and Stokes factors. Preprint 2008. arXiv:0801.3974

[2] J. Engel, M. Reineke: Smooth models of quiver moduli. Preprint 2007. To appear in Math. Z. arXiv:0706.4306

[3] M. Gross, R. Pandharipande, B. Siebert: The tropical vertex. Preprint 2009. arXiv:0902.0779

[4] M. Kontsevich, Y. Soibelman: Stability structures, Donaldson-Thomas invariants and cluster transformations. Preprint 2008. arXiv:0811:2435

[5] M. Reineke: Feigin's map and monomial bases for quantized enveloping algebras. Math. Z. 237 (2001) 3, 639-667.

[6] M. Reineke: The Harder-Narasimhan system in quantum groups and cohomology of quiver moduli. Invent Math. 152 (2003), 349-368.

[7] M. Reineke: Moduli of representations of quivers. In: Trends in Representation Theory of Algebras and Related Topics (ed. A. Skowronski), EMS Series of Congress Reports, EMS Publishing House, 2008. arXiv:0802.2147 